\title{
Nanostructure of the Iron Chalcogenide Superconductor $\mathrm{Fe}_{1+\mathrm{y}} \mathrm{Te}_{\mathrm{x}} \mathrm{Se}_{1-\mathrm{x}}$
}

\author{
Hefei $\mathrm{Hu}^{1,3}$, Jian-Min Zuo ${ }^{2,3,5}$, Jinsheng $\mathrm{Wen}^{4}$, Zhijun $\mathrm{Xu}^{4}$, Zhiwei $\mathrm{Lin}^{4}$, Qiang $\mathrm{Li}^{4}$, Genda $\mathrm{Gu}^{4}$, Wan \\ Kyu Park ${ }^{1,3}$ and Laura H Greene ${ }^{1,3}$ \\ ${ }^{1}$ Department of Physics, University of Illinois at Urbana-Champaign, Urbana, Illinois 61801 \\ ${ }^{2}$ Department of Materials Science and Engineering, University of Illinois at Urbana-Champaign, \\ Urbana, Illinois 61801 \\ ${ }^{3}$ Frederick Seitz Materials Research Laboratory, University of Illinois at Urbana-Champaign, \\ Urbana, Illinois 61801 \\ ${ }^{4}$ Condensed Matter Physics and Materials Science Department, Brookhaven National Laboratory, \\ Upton, New York 11973
}

High- $\mathrm{T}_{\mathrm{c}}$ superconductors have great potential in energy saving for real world applications, such as power transmission lines, electric motors, maglev trains, and etc. Discovery of the high- $\mathrm{T}_{\mathrm{c}}$ ironbased superconductors, such as $\mathrm{LaFeAsO}_{1-\mathrm{x}} \mathrm{F}_{\mathrm{x}}[1]$, have attracted renewed interest in high- $\mathrm{T}_{\mathrm{c}}$ superconductors. Among the iron-based superconductors synthesized so far, 11- type $\mathrm{Fe}_{1+\mathrm{y}} \mathrm{Te}_{\mathrm{x}} \mathrm{Se}_{1-}$ ${ }_{x}[2]$ has the simplest crystal structure, comprised of FeTe/Se layers only, which is ideal for investigating the underlying mechanism in superconductivity. Investigation on the structure of $\mathrm{Fe}_{1+\mathrm{y}} \mathrm{Te}_{\mathrm{x}} \mathrm{Se}_{1-\mathrm{x}}$ is essential to further understand the properties of this material. Scanning transmission electron microscopy(STEM) and electron energy loss spectroscopy(EELS) are applied to examine crystal and electronic structures of $\mathrm{Fe}_{1+\mathrm{y}} \mathrm{Te}_{\mathrm{x}} \mathrm{Se}_{1-\mathrm{x}}$ single crystals.

In STEM, incoherently scattered intensities, collected by the high angle annular dark field (HAADF) detector with a large inner cutoff angle, are proportional to atomic numbers. Fig. 1 shows HAADF images at low magnification of (a) $\mathrm{Fe}_{1.07} \mathrm{Te}_{0.75} \mathrm{Se}_{0.25}$, (b) $\mathrm{Fe}_{1.08} \mathrm{Te}_{0.55} \mathrm{Se}_{0.45}$, (c) $\mathrm{FeTe}_{0.7} \mathrm{Se}_{0.3}$ and (d) $\mathrm{FeTe}_{0.55} \mathrm{Se}_{0.45}$, along the $\mathrm{c}$ axis. The images show clear interconnected, fractal-like, contrast for all of the samples. The brighter regions contain higher Te concentration than the darker regions, since Te atoms are heavier than Se atoms. EELS can be recorded simultaneously in STEM mode. The characteristic energy of core loss edges in EELS, resulted from inner-shell ionization, provides direct chemical information. Quantitative analysis on Te content and energy-loss near-edge structure (ELNES) of the Fe- $\mathrm{L}_{2,3}$ edge, especially the $\mathrm{L}_{3} / \mathrm{L}_{2}$ white-line intensity ratio, are studied by the STEM-EELS technique. Fig. 2(a) shows a HAADF image of $\mathrm{FeTe}_{0.7} \mathrm{Se}_{0.3}$ with a line demonstrating the path along which EELS spectra were recorded. A typical spectrum is shown in Fig. 2(b). The integrated Te-M $\mathrm{M}_{4,5}$ intensity along the scanning path after background subtraction is shown as the red curve in the Fig. 2(c). The change in the integrated intensity indicates that the fluctuation of $\mathrm{Te}$ concentration along the scan by $20 \%$ or more. The intensity ratio of the $\mathrm{Fe} \mathrm{L}_{3}$ and $\mathrm{L}_{2}$ edge along the EELS scan was also calculated, shown as the black curve in the Fig. 2(c). The change in the $\mathrm{L}_{3} / \mathrm{L}_{2}$ intensity ratio shows a clear correlation with the Te content fluctuation. These results demonstrate direct evidences of phase separation in $\mathrm{Fe}_{1+\mathrm{y}} \mathrm{Te}_{\mathrm{x}} \mathrm{Se}_{1-\mathrm{x}}$ single crystals and changes in the d-electron states at the nanometer scale. Nanostructures of Oxygen annealed samples will be also discussed.

References

[1] Y. Kamihara et al. JACS 130 (2008) 3296-3297

[2] K. W. Yeh et al. EPL 84 (2008) 37002 


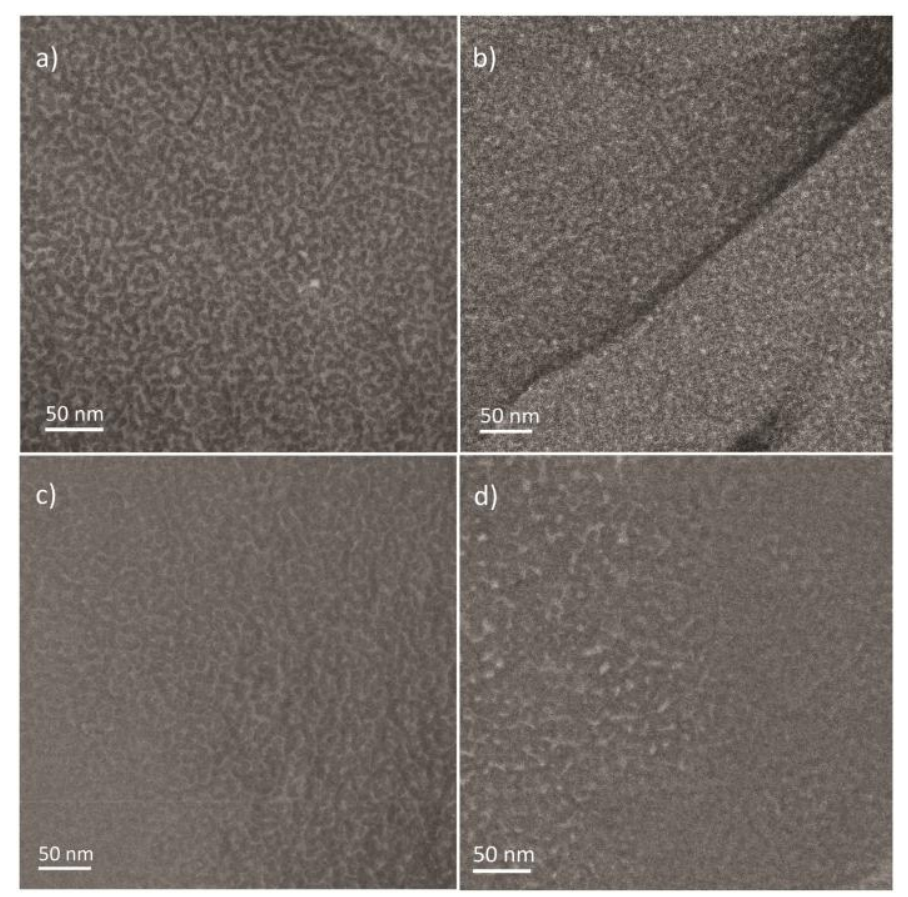

Figure 1. HAADF images at low magnification sample (a) $\mathrm{Fe}_{1.07} \mathrm{Te}_{0.75} \mathrm{Se}_{0.25}$, (b) $\mathrm{Fe}_{1.08} \mathrm{Te}_{0.55} \mathrm{Se}_{0.45}$, (c) $\mathrm{FeTe}_{0.7} \mathrm{Se}_{0.3}$ and (d) $\mathrm{FeTe}_{0.55} \mathrm{Se}_{0.45}$.
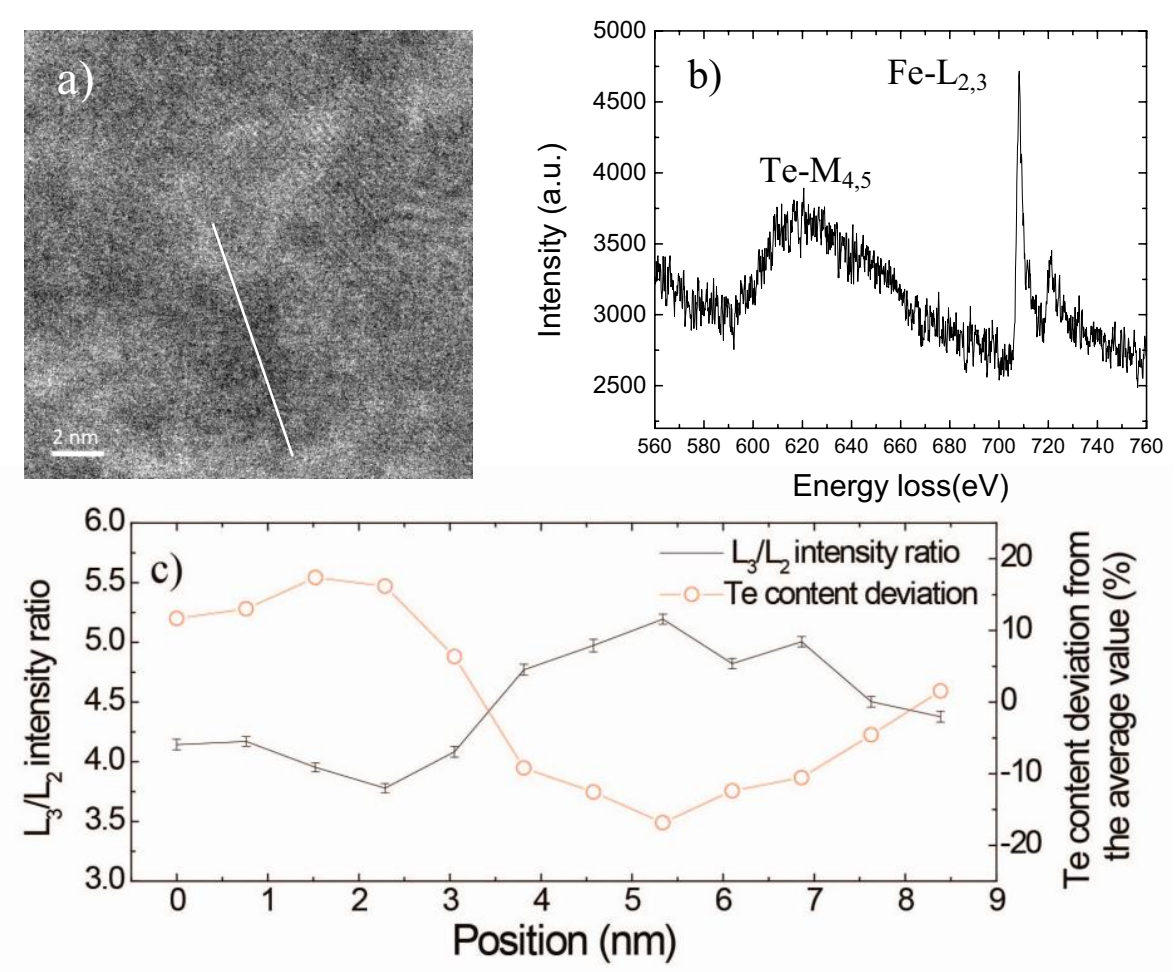

Figure 2. (a) A HAADF image of $\mathrm{FeTe}_{0.7} \mathrm{Se}_{0.3}$ with a line demonstrating the path along which EELS spectra were recorded. (b) a typical energy loss spectrum. (c) The red and black curves show variation of Te-M $\mathrm{M}_{4,5}$ intensity and $\mathrm{L}_{3} / \mathrm{L}_{2}$ white-line intensity ratio along the EELS scan, respectively. 